\title{
Stem Cell Therapy for Sensorineural Hearing Loss, Still Alive?
}

\author{
Yong-Ho Park \\ Department of Otolaryngology-Head and Neck Surgery, Brain Research Institute, College of Medicine, Chungnam National University, \\ Daejeon, Korea
}

\section{Received August 14, 2015 \\ Revised August 15, 2015 \\ Accepted August 18, 2015}

\section{Address for correspondence}

Yong-Ho Park, MD, PhD

Department of Otolaryngology-

Head and Neck Surgery,

Brain Research Institute,

College of Medicine,

Chungnam National University,

282 Munhwa-ro, Jung-gu,

Daejeon 35015, Korea

Tel +82-42-280-7697

Fax +82-42-253-4059

E-mail parkyh@cnu.ac.kr
In mammals, the auditory system, which includes the cochlea, has a very complex structure harboring many types of cells performing different functions. Among these cells are the auditory hair cells (HCs), which are terminally and well differentiated unique cells which have lost their regenerative potential after development. The auditory HCs are easily damaged by aging as well as during episodes of ototoxicity and acoustic trauma. HCs damages typically occur in the early stage of injury and can result a permanent hearing loss. Recently, there have been tremendous developments from stem cells (SCs) research involving sensorineural hearing loss, but several limitations and obstacles persist in allowing these developments from continuing onto clinical applications. This review discusses the recent advances in SC research in sensorineural hearing loss with the subsequent sections discussing the possible hurdles and limitations that currently preclude their clinical application.

J Audiol Otol 2015;19(2):63-67

\section{Introduction}

Successful treatment or rehabilitation of severe to profound sensorineural hearing loss can be achieved with hearing aids and various implantable hearing devices including cochlear implants. These options are well known and available worldwide but are not suitable or applicable for all patients. Some patients are even unsatisfied with using these devices. Since the description of stem cells (SCs) or stemness in the mammalian cochlea [1], trials of inducing regeneration or trans-differentiation of these SCs using various methods have attracted attention towards their eventual application in affected patients [2-18].

If there are cells in the cochlea which have a potential to differentiate, their trans-differentiation would be more suitable for managing sensorineural hearing loss. If there are no cells capable of differentiation in the cochlea, then SCs would be feasible for treatment. There have been outstanding progresses in these areas of research. The creation of inner ear

This is an Open Access article distributed under the terms of the Creative Commons Attribution Non-Commercial License (http://creativecommons. org/licenses/by-nc/3.0/) which permits unrestricted non-commercial use, distribution, and reproduction in any medium, provided the original work is properly cited. progenitor cells from murine embryonic SCs in vitro and integrating these progenitor cells into the developing inner ear at sites of epithelial injury expressing the $\mathrm{HC}$ markers has been reported [19]. Auditory hair cells (HCs) can be replaced and hearing improved by Atoh1 gene therapy in deaf mammals [20]. HC-like cells with mechano-sensitive functioning cilia can be generated from embryonal SCs (ESCs) and inducible pluripotent SCs [21]. More recently, generation of functioning inner ear sensory epithelia from pluripotent SCs was reported [22]. Transplantation of human ESCs derived otic progenitors into the spiral ganglion (SG) resulted in these cells surviving with functional recovery in animal affected by auditory neuropathy [23].

There are still many more obstacles preventing the clinical application of the aforementioned methods for sensorineural hearing loss in addition to the already known basic problems associated with SCs such as tumor formation, graft or transplantation failure and immune-rejection. The difficulties with managing inner ear conditions can be briefly classified into host factors (intracochlear) and extrinsic factors. The very complex structure of the cochlea and it being located in the center of the skull pose significant limitations with regards to it being properly assessed. The next sections will discuss the 
limitations or possible hurdles that preclude the clinical application of SCs in sensorineural hearing loss. Also included are review of related recent research and suggestions on what should be done to safely apply SCs in the management of sensorineural hearing loss.

\section{Review}

\section{Target area in the cochlea}

As described before, the cochlea, which is located deep inside the skull, has a very complex structure and harbors many different types of cells. The different types of cells or different cochlear areas can be targeted for SC application. For example, if the hearing loss is because of auditory HCs involvement, then the target of treatment should be the HCs and if the hearing loss is due to a lateral cochlear wall pathology, then the target should be directed at the lateral cochlear wall. Almost all hearing loss, except for some due to genetic origin, are associated with HCs and nerve fibers involvement, so the fibers and $\mathrm{HCs}$ would be the main targets for SC therapy (Fig. 1).

Auditory HCs loss is well known initial and early histopathologic finding in hearing loss. Numerous reports have been made about auditory HCs loss in noise injury, ototoxic drugs, and aging process. But HCs loss is limited only in the early stage of hearing loss and almost all hearing loss, over time, gradually result in loss of supporting cells in the auditory epithelium, followed by cochlear nerve and SG neuronal degeneration.

Second, although the SG neuron (SGN) can be a target for SCs application in hearing loss, hearing cannot be restored with an intact SGN only. Furthermore, only a few cases of hearing loss are due to just SG neuronal or nerve fiber problems. This means that in cases of hearing loss wherein SG neuronal loss and nerve fiber problem have been detected, an underlying $\mathrm{HC}$ problem should still be considered since the $\mathrm{HC}$ has a role at the initial or terminal structure of hearing.

Third, the lateral wall of the cochlea which is composed of the stria vascularis and spiral ligament can be a target for SC treatment in diseases that show histopathologic abnormalities in the lateral wall of the cochlea.

Other areas showed in Fig. 1 may be targeted for SC treatment in some cases of hearing loss of unknown origin. The problem with choosing a target area in the cochlea for SC therapy is that even though we can evaluate or estimate the hearing level and severity of hearing loss in affected patients through various audiologic tests, these tests cannot reliably reflect the actual histopathologic finding in the ear of these patients. It is impossible to determine the precise cochlear

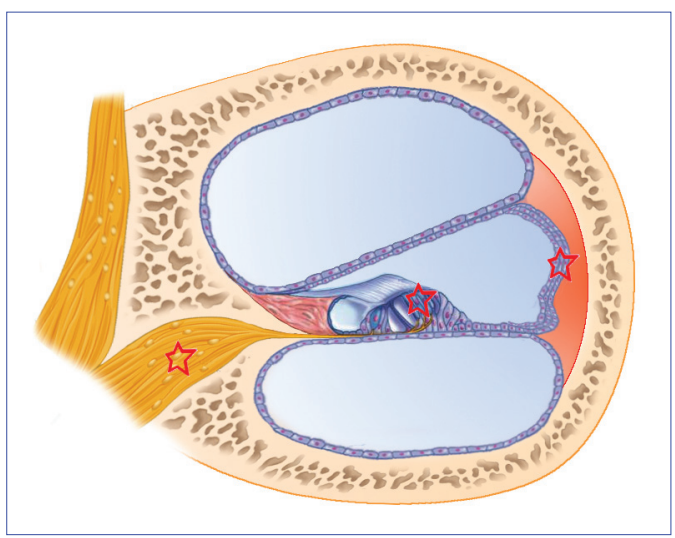

Fig. 1. Potentially target area of SCs in the cochlea (综). Variable areas such as auditory epithelium, SGN and stria vascularis can be a target for SC applications. SGN: spiral ganglion neuron, SCs: stem cells.

pathology without a biopsy or autopsy.

\section{Types of SCs}

The choice of species or developmental stage of the SCs that will be used for transplantation can be decided depending on the target area (HCs, SGN, etc.) if the details of the histopatholgy are known.

First, the species of SCs should be considered. For example, neural SCs will be more suitable in the management of SGN or nerve fibers pathology. Recently Chen, et al. [23] reported the restoration of auditory evoked responses using embryonic stem cell derived otic porgenitors. Other neural SCs may be more applicable for SGN replacement [9,12,14,24]. The auditory epithelium, HC-like cells or SCs which have a potential to differentiate into HCs may be used, although questions still exist whether these cells are enough to replace damaged HCs and other cells in the auditory epithelium including various types of supporting cells (e.g., Deiter's, Pilla, Claudius, Hensen's etc.). More recently, there was a report about generation of inner ear sensory epithelia from pluripotent SCs using 3D culture method [22].

Second, the developmental stages (differentiated or undifferentiated) of SCs should be considered. For example, a decision should be made whether to transplant totipotent cells to nullpotent cells. In other words, whether to transplant already well differentiated targeted cells or partly or undifferentiated cells that will, hopefully, differentiate into the desired cells in the target area. One should remind that the HCs in the auditory epithelium are divided into the inner HCs and outer HCs which perform different functions. Moreover, these cells are quite different from the apex to the base and also show differences from the first to the third low (Fig. 2). It seems that these differences are not limited to the HCs only. It may be safe to assume that two cells are not alike in the auditory epi- 


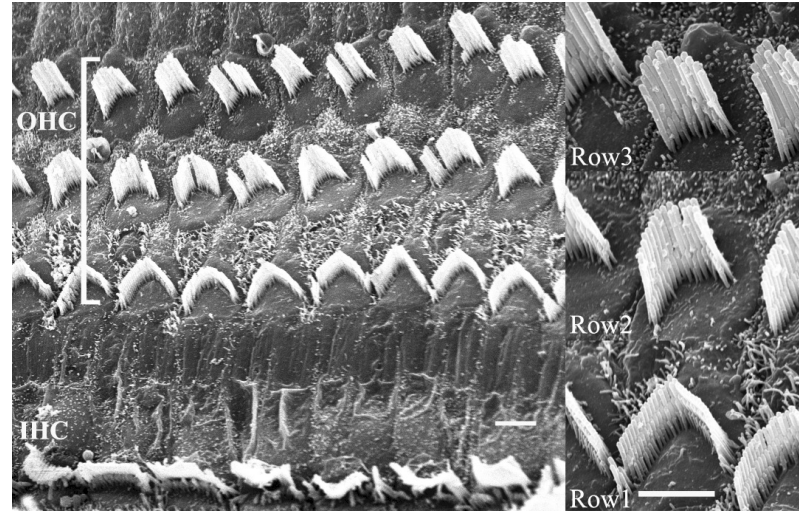

Fig. 2. Differences of HCs in the auditory epithelium. Stereocilia of $\mathrm{HCs}$ is quite different in $\mathrm{IHC}$ and $\mathrm{OHC}$ and even in the $\mathrm{OHC}$ according to the row. $\mathrm{HCs}$ : hair cells, $\mathrm{IHC}$ : inner $\mathrm{HC}, \mathrm{OHC}$ : outer HC. Scale bar $=4 \mu \mathrm{m}$.

thelium or in the cochlea as a whole. Transplantation of undifferentiated or partly differentiated cells and then inducing them to differentiate into the desired cells in the appropriate target area surrounded by supporting cells seem to be more feasible. Inducing cell differentiation may be possible since delta-notch, wnt, and lateral inhibitions between HCs and supporting cells are well known signals in cochlear and $\mathrm{HC}$ development. Using these signals should be considered in the directed differentiation of transplanted cells. So far, induced differentiation has only been towards HC-like cells. The above technique may not be applicable for the other possible target areas of the cochlea.

\section{Transplantation method (how and where)}

Transplantation of cells into the cochlea is challenging because the cochlea is positioned deep inside of the skull and surrounded by hard bony structures. The cochlea is further partitioned into the scala tympani, media and vestibule. Even though surgically accessing the cochlea through the cochleostomy or round window approaches have been widely used for cochlear implantation surgery, the surgical process can still cause disturbance or disruption of cochlear homeostasis that can result in residual hearing loss and vertigo. The surgical techniques mentioned above are also limited to accessing just the scala tympani in the basal turn of the cochlea. It is also doubtful whether the auditory HCs that were transplanted into the scala tympani will migrate into the scala media and other target areas such as the auditory epithelium through the basilar membrane or helicotrema. In addition, the detailed pattern of lymphatic flow in the cochlea is still unknown. Although there was a report about embryonic stem cells being transplanted through the basilar membrane and survived in the scala media [8], there was no evidence of the transplanted cells integrating into the cochlear structure and there are still questions on how the cells could survive in the scala media. More recently there is a report showing that cells transplanted onto the scala media by a direct approach through the lateral wall were able to survive [25]. Although SC transplantation into the SG in animal model have been reported $[12,14,17,23]$, in clinical perspective it seems more difficult to assess the status of the SGN or auditory nerve than the cochlea. The number of cells to be transplanted at such target area are also unknown. This will be discussed in the next section.

\section{Enhancing cell survival in the cochlea after transplantation}

To date, none have reported the survival rate of cells transplanted into the cochlea. As described before, the insides of the cochlea is subdivided into three spaces: scala tympani, media and vestibuli. The scala tympani and vestibuli are filled with perilymph which has low potassium $(\sim 10 \mathrm{mEq} / \mathrm{L})$ and high sodium $(\sim 140 \mathrm{mEq} / \mathrm{L})$ concentration, similar to extracellular fluids. The scala media, on the other hand, is filled with high potassium $(\sim 144 \mathrm{mEq} / \mathrm{L})$ and low sodium $(\sim 5$ $\mathrm{mEq} / \mathrm{L}$ ) endolymph, somewhat similar to intracellular fluids. These differences in ion concentrations are important in maintaining endocochlear potential and cochlear homeostasis. Unfortunately, no cells, including SCs, will survive in this high potassium condition. It is safe to expect that transplanted cells or cells migrating into the scala media will not survive easily. This is one of the main hurdle for SCs transplantation in the cochlea especially when targeting the auditory epithelium or other structures located within the scala media. An experiment has already shown that cells did not survive in an artificial endolymph [25]. Finding ways to ensure survival of the transplanted cells, especially when targeting the auditory epithelium, is currently a major undertaking. Park, et al. [25] revealed that transplanted cells can survive in the scala media by flushing the endolymph with artificial perilymph in an experimental animal model, but this method of flushing the endolymph to reduce the high potassium content in the scala media appears difficult to do in humans. Moreover there is still no data or report on cell survival rate or survival time inside the cochlea. Although it is known that around 3500 inner HCs and 12000 outer HCs exist in humans, the number of transplanted cells in relation to survival rate will be needed. The issues involved around dealing with SG pathology are even more complex. It may be nearly impossible to assess all of the SGNs, and the possible migration of transplanted cells in the SGN to the other SGNs is still unknown.

\section{Integration into appropriate area}

Transplanted cell integration into the target area looks 
more feasible in the SG than in the auditory epithelium because the inter-cellular junctions in the auditory epithelium are composed of tight junctions and adherence junctions, inter-cellular junctions that serve as barriers against integration or incorporation of the transplanted cells into the auditory epithelium. Various methods of inter-cellular junctional modulation may be able to overcome these barriers and a report has shown that transient junctional disruption could be induced with sodium caprate which is already widely used to increase intestinal drug absorption through the paracellular route. They showed that sodium caprate could transiently disrupt the auditory epithelial junctions and may aid in the integration of transplanted cells into the auditory epithelium. But successful integration of transplanted cell into the appropriate area (i.e., the area where the original $\mathrm{HC}$ was located) cannot be guaranteed. To complicate matters further, the basilar membrane, on which the auditory epithelium is located, differs from the apex to the base in terms of thickness and width.

\section{Correct positioning (direction) ?}

The HCs in the auditory epithelium have cilia where the mechano-electric transition first occurs. When the cilia move in synchrony toward the basal body, an influx of potassium through the tip occurs and then the HCs are excited. It is still unknown whether the transplanted or differentiation induced HCs would orient themselves in the normal position (Fig. 2). If the transplanted cells have integrated or developed as shown in Fig. 3, the entire system would not function properly since the cilia would move asynchronously causing some HCs to be inhibited while others are excited.

\section{Synaptogenesis between HCs and nerve}

Hearing can not be obtained with auditory HCs' role of mechano-electric transition functioning in isolation. Transferring of the generated electric signal to the auditory nerve and central nervous system is mandatory. Successfully transplanted cells in the auditory epithelium will be useless if synaptogenesis between HCs and nerve fibers does not occur. The synapse is a very complex structure wherein afferent or efferent fibers should be considered along with the different cell types (such as outer HCs or inner HCs) that they will be coupled with (Fig. 4). Recent studies have been done on nerve regeneration using various methods and any advancement achieved in this area will definitely be a step towards developing techniques of combining them with SCs transplantation [26,27].
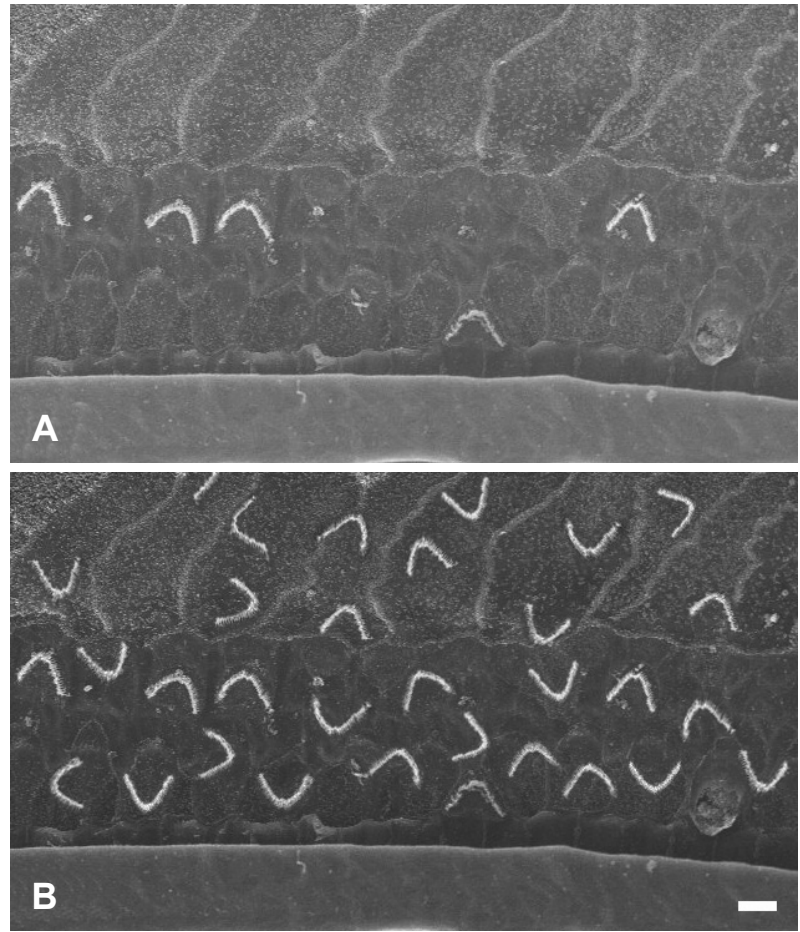

Fig. 3. Directions of HCs in the auditory epithelium. A: Damaged auditory epithelium. B: Virtual image of SC transplanted or transdifferentiated auditory epithelium. Scale bar $=4 \mu \mathrm{m}$. SC: stem cell, HCs: hair cells.

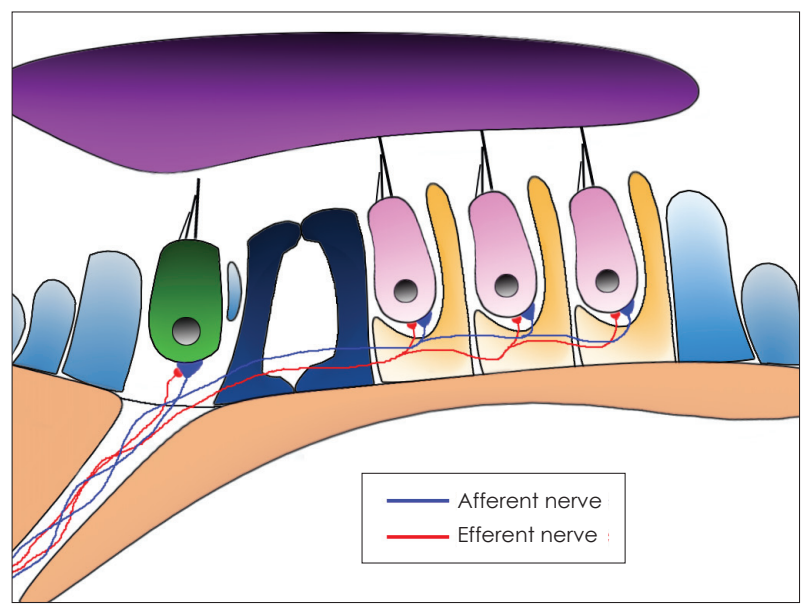

Fig. 4. Afferent and efferent fibers in the HCs. HCs: hair cells.

\section{Conclusion}

SCs technology is feasible for repairing or replacing organs and cells which have no regenerative potential but a lot of obstacles preclude its use in the management of hearing loss. However, when combined with gene therapy or trans-differentiation, the use of SCs for hearing loss may be possible. Ask me whether SCs in hearing loss is possible, I would say "It is not easy, but it is not impossible". 


\section{Acknowledgments}

I thank Kwan-Ho Kim and Ah-Ra Lyu for their beautiful illustrations. This work was supported by Chungnam National University Hospital Research Fund, 2013.

\section{REFERENCES}

1) Li H, Liu H, Heller S. Pluripotent stem cells from the adult mouse inner ear. Nat Med 2003;9:1293-9.

2) Ishimoto S, Kawamoto K, Kanzaki S, Raphael Y. Gene transfer into supporting cells of the organ of Corti. Hear Res 2002;173:187-97.

3) White PM, Doetzlhofer A, Lee YS, Groves AK, Segil N. Mammalian cochlear supporting cells can divide and trans-differentiate into hair cells. Nature 2006;441:984-7.

4) Batts SA, Raphael Y. Transdifferentiation and its applicability for inner ear therapy. Hear Res 2007;227:41-7.

5) Izumikawa M, Batts SA, Miyazawa T, Swiderski DL, Raphael Y. Response of the flat cochlear epithelium to forced expression of Atoh1. Hear Res 2008;240:52-6.

6) Vlastarakos PV, Nikolopoulos TP, Tavoulari E, Papacharalambous G, Tzagaroulakis A, Dazert S. Sensory cell regeneration and stem cells: what we have already achieved in the management of deafness. Otol Neurotol 2008;29:758-68.

7) Lin V, Golub JS, Nguyen TB, Hume CR, Oesterle EC, Stone JS. Inhibition of Notch activity promotes nonmitotic regeneration of hair cells in the adult mouse utricles. J Neurosci 2011;31:15329-39.

8) Hildebrand MS, Dahl HH, Hardman J, Coleman B, Shepherd RK, de Silva MG. Survival of partially differentiated mouse embryonic stem cells in the scala media of the guinea pig cochlea. J Assoc Res Otolaryngol 2005;6:341-54.

9) Parker MA, Corliss DA, Gray B, Anderson JK, Bobbin RP, Snyder EY, et al. Neural stem cells injected into the sound-damaged cochlea migrate throughout the cochlea and express markers of hair cells, supporting cells, and spiral ganglion cells. Hear Res 2007;232:29-43.

10) Kamiya K, Fujinami Y, Hoya N, Okamoto Y, Kouike H, Komatsuzaki R, et al. Mesenchymal stem cell transplantation accelerates hearing recovery through the repair of injured cochlear fibrocytes. Am J Pathol 2007;171:214-26.

11) Ulfendahl M, Hu Z, Olivius P, Duan M, Wei D. A cell therapy approach to substitute neural elements in the inner ear. Physiol Behav 2007;92:75-9.

12) Matsuoka AJ, Kondo T, Miyamoto RT, Hashino E. Enhanced survival of bone-marrow-derived pluripotent stem cells in an animal model of auditory neuropathy. Laryngoscope 2007;117:1629-35.

13) Altschuler RA, O'Shea KS, Miller JM. Stem cell transplantation for auditory nerve replacement. Hear Res 2008;242:110-6.
14) Lang H, Schulte BA, Goddard JC, Hedrick M, Schulte JB, Wei L, et al. Transplantation of mouse embryonic stem cells into the cochlea of an auditory-neuropathy animal model: effects of timing after injury. J Assoc Res Otolaryngol 2008;9:225-40.

15) Jongkamonwiwat N, Zine A, Rivolta MN. Stem cell based therapy in the inner ear: appropriate donor cell types and routes for transplantation. Curr Drug Targets 2010;11:888-97.

16) Han Z, Yang JM, Chi FL, Cong N, Huang YB, Gao Z, et al. Survival and fate of transplanted embryonic neural stem cells by Atoh1 gene transfer in guinea pigs cochlea. Neuroreport 2010;21:490-6.

17) Cho YB, Cho HH, Jang S, Jeong HS, Park JS. Transplantation of neural differentiated human mesenchymal stem cells into the cochlea of an auditory-neuropathy guinea pig model. J Korean Med Sci 2011;26:492-8.

18) Okano T, Kelley MW. Stem cell therapy for the inner ear: recent advances and future directions. Trends Amplif 2012;16:4-18.

19) Li H, Roblin G, Liu H, Heller S. Generation of hair cells by stepwise differentiation of embryonic stem cells. Proc Natl Acad Sci U S A 2003;100:13495-500.

20) Izumikawa M, Minoda R, Kawamoto K, Abrashkin KA, Swiderski DL, Dolan DF, et al. Auditory hair cell replacement and hearing improvement by Atoh1 gene therapy in deaf mammals. Nat Med 2005; 11:271-6.

21) Oshima K, Shin K, Diensthuber M, Peng AW, Ricci AJ, Heller S. Mechanosensitive hair cell-like cells from embryonic and induced pluripotent stem cells. Cell 2010;141:704-16.

22) Koehler KR, Mikosz AM, Molosh AI, Patel D, Hashino E. Generation of inner ear sensory epithelia from pluripotent stem cells in 3D culture. Nature 2013;500:217-21.

23) Chen W, Jongkamonwiwat N, Abbas L, Eshtan SJ, Johnson SL, Kuhn S, et al. Restoration of auditory evoked responses by human ES-cell-derived otic progenitors. Nature 2012;490:278-82.

24) Wang Y, Dong MM. In vitro induction and differentiation of newborn guinea pig hippocampus neural stem cells into cells resembling inner hair cells, using artificial perilymph. J Laryngol Otol 2011; 125:771-5.

25) Park YH, Wilson KF, Ueda Y, Tung Wong H, Beyer LA, Swiderski $\mathrm{DL}$, et al. Conditioning the cochlea to facilitate survival and integration of exogenous cells into the auditory epithelium. Mol Ther 2014; 22:873-80.

26) Duan M, Venail F, Spencer N, Mezzina M. Treatment of peripheral sensorineural hearing loss: gene therapy. Gene Ther 2004;11 Suppl 1:S51-6.

27) Shibata SB, Cortez SR, Beyer LA, Wiler JA, Di Polo A, Pfingst $\mathrm{BE}$, et al. Transgenic BDNF induces nerve fiber regrowth into the auditory epithelium in deaf cochleae. Exp Neurol 2010;223:464-72. 